\title{
Grid Services for Derivatives Pricing
}

\section{Jürgen Schumacher}

$C \& C$ Research Laboratories, NEC Europe Ltd. and Dept. of Computer Science III, University of Bonn Rathausallee 10, D-53757 Sankt Augustin, Germany

E-mail: schumacher@ccrl-nece.de

\section{Uwe Jaekel}

C\&C Research Laboratories, NEC Europe Ltd.

Rathausallee 10, D-53757 Sankt Augustin, Germany

E-mail:jaekel@ccrl-nece.de

\section{Falk Zimmermann}

C\&C Research Laboratories, NEC Europe Ltd.

Rathausallee 10, D-53757 Sankt Augustin, Germany

E-mail: zimmermann@ccrl-nece.de

The growth of derivatives markets combined with the rising complexity of the issued products and stricter regulatory requirements are leading to an increased demand for compute power. At the same time, derivatives valuation systems and many other financial applications are forced to support shorter financial products life-cycles, real-time valuation and risk management in a cost effective, secure and scalable way. We have developed a simulation based derivatives valuation toolkit for the parallel valuation of multi-dimensional European-, Bermudian- and Americanstyle options which can be easily extended with new product functions. This application has been deployed as a valuation service within the GEMSS grid infrastructure, enabling the secure invocation from a Microsoft Excel client of distinct derivatives valuation services of internal or external service providers. The experiences made with this prototype are used in the NextGRID project for the definition of requirements for next generation Grid architectures adapted to business application needs and the construction of revised Grid enabled applications for derivatives valuation and robust portfolio optimization.

Grid Technology for Financial Modeling and Simulation

February 3/4, 2006 - Palermo, (Italy)

\section{${ }^{*}$ Speaker}




\section{Introduction}

With the growth of derivatives markets, accompanied by an increase in both the diversity and the complexity of products such as Bermudian options on multiple risk factors, the valuation of derivatives products has become an important part of the overall IT process in a financial institution. The requirements for the derivatives valuation infrastructure are demanding in several respects. The infrastructure has to make optimal use of administrative, computing, data and network resources. It has to deliver derivative prices and sensitivities with high accuracy at short notice in order to prevent ineffective risk management, attributable to outdated or unreliable results, or customers moving to faster competitors. The system has to adapt quickly to trade volume increases to avoid limiting trading activity. Furthermore it should facilitate the integration of new derivatives products since these products can usually be sold at higher margins before they become common place. In contrast to these requirements, current derivative valuation infrastructures are often contained in monolithic software systems, requiring exclusive hardware. In most cases these systems can neither be adapted quickly and cost effectively to changes in trade volumes nor to new products. In order to study to which extent the stated requirements can be satisfied by the integration of derivatives valuation algorithms with Grid technology, we have developed a Grid prototype system which integrates derivatives valuation services based on our simulation based valuation toolkit and makes them available to Excel clients. The prototype is based on the GEMSS grid infrastructure, which was originally developed for medical services.

\section{The derivative pricing application}

For the realization of the derivative pricing service we have developed a portable simulation based pricing toolkit that enables the estimation of derivatives prices and sensitivities for European- and American/Bermudian-style options. The toolkit allows the parallel valuation of European style derivatives by Monte-Carlo and Quasi-Monte Carlo simulation as well as the parallel valuation of Bermudian style derivatives by the Stochastic Mesh approach of Broadie and Glasserman [1,2,3]. The simulation toolkit has been tested on a variety of different architectures ranging from NEC-SX vector computers to PC-Clusters, providing a set of basic random number and low-discrepancy generators, underlying models and product templates. It is designed to be easily extendable to new product templates and underlying models, which can be integrated internally or invoked by the toolkit.

\section{The GEMSS Grid middleware}

The GEMSS architecture [4,5] uses a client/server topology employing a service-oriented architecture basing on standard web service (WS) technology. As in the medical world, security is of central importance in the finance community. Clearly, adoption of technology will only happen if a sufficient level of security can be demonstrated. The GEMSS infrastructure uses Tomcat with a plug-in to implement WS security avoiding possible shortfalls of standard technologies like HTTPS. It implements an end-to-end security framework deployed within the context of a public key infrastructure on top of transport level HTTPS. End-to-end security is particularly valuable when the underlying network topology includes intermediaries, such as 
web servers in "demilitarized" zones. Together with the Transport and Messaging Framework and basic message-level security it ensures a high degree of protection for financial data processed by GEMSS. Reservation based schedulers are supported and quality of service code is provided to allow reservations to be made in advance of the job start time. Application specific performance modeling is used to estimate the quality of service requirements for specific jobs. Negotiation code allows clients to get the best deal from multiple service providers, all offering the service capabilities from which the clients choose.

The client is the active component driving the three-stage resource allocation process: the discovery of suitable services, the request for offers for a defined task and finally the selection of a suitable service provider. This active role of the client is required in order to operate with firewalls and not tunnel through them. Given this, there can be no service provider initiated connections, or call-backs, to the client. Applications will be run by the service on resources available at the service provider site under the control of the client, subject to the necessary availability of and authority to use resources. A quality of service management module handles reservations with the compute resource job scheduler and provides input to the quality of service negotiation process so that sensible bids can be made to client job requests. The QoS management module requires an application specific performance module to estimate the execution time jobs will take, given a meta data description of the job's input data.

For transforming a native GEMSS application into a Web Service, a generic Java component, called ApplicationService, is utilized which provides generic methods for uploading input data, starting a job, downloading output-data, and for status queries. These methods are exposed via a WSDL document that is generated from a corresponding Java interface. An intuitive graphical user interface has been developed in order to enable users to specify application descriptors without having to cope with the details of XML.

\section{Results and Conclusion}

The current Grid prototype permits the secure invocation from Excel user clients of the derivatives pricing service, which can be hosted either by an internal department or by an external service provider. The GEMSS system enables the prototype to be operated safely on top of typical network systems with firewalls on the client and the service provider site without requiring changes to the network security standards. The derivatives valuation problems can be defined in the current Excel client and submitted to the valuation service by a call to the VBA API for the Grid functions. The implementation of the API handles then the interaction with the GEMSS client by which the service requests, offer confirmation, job submission, execution observation and result collection is performed. After the job has been finished the results are returned to the Excel client where they can be used in further computations of the spreadsheet or the invoked VBA application. The simulation based valuation services deployed in the prototype shows near linear scalability for European and American style valuation problems and moderate cluster sizes. Some performance results for a European and a Bermudian basket option for different number of processors and architectures are shown in Figure 1. The execution times shown are the computation times after invocation. Within the current implementation the system takes between 30s and 1 minute for the Grid interactions. 


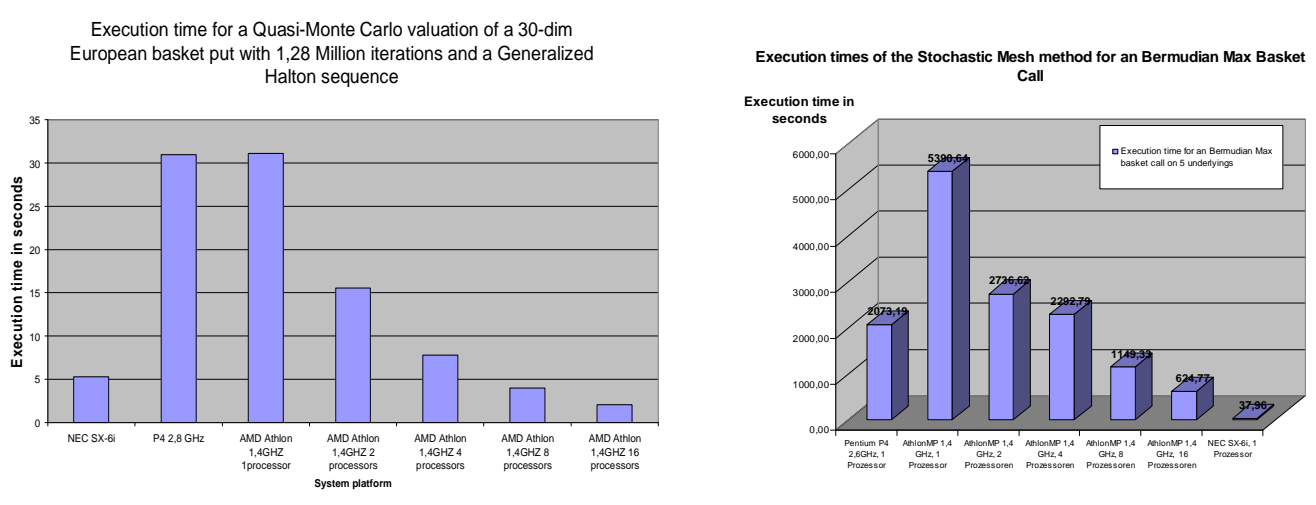

Figure 1: Scalability of the derivatives pricing service

Although the underlying system already provides a lot of useful features, which ease the deployment of Grid systems in financial institutions, it has to be improved in order to be widely applicable. Therefore, the collaborative activities linked to derivatives pricing within the NextGRID project address:

- The improved integration of important legacy software like Microsoft Excel, which is slow in the current prototype.

- The development of new service level based business models for the interaction between internal and external resource provider organisations and the different user types in financial institutions that are better suited for financial applications and provide less overhead than the current request/offer based system.

- The reduction of service invocation latency, which can be prohibitive for small problem sizes, with the help of an interactive resource usage mode and the NextGRID dynamic security model in order to make the pricing service usable for front office applications.

The investigations of the above are being used to influence the direction of architectural and middleware developments in the NextGRID project.

\section{Acknowledgements:}

This work was partly supported by the European Commission's IST research program under the contracts IST-2001-37153 GEMSS and 511563-NextGRID. This paper expresses the authors' opinions and not necessarily those of the European Commission. The Commission is not liable for any use that may be made of the information contained in this document.

\section{References}

[1] J. Schumacher, U. Jaekel, A. Basermann, Parallelization and Vectorization of Simulation Based Option Pricing Methods, ICCSA 03 (2003), pp 139-147.

[2] P. Boyle, Options: A Monte Carlo Approach, J. of Financial Economics 4,4, 1977, 323-338.

[3] M. Broadie, P. Glasserman, A Stochastic Mesh Method for Pricing High-Dimensional American Option, Journal of Computational Finance, Vol 7, 4, 2004, 35-72.

[4] S. Benkner, G. Berti, G. Engelbrecht, J. Fingberg, G. Kohring, S.E. Middleton, and R. Schmidt. GEMSS: grid infrastructure for medical service provision. Methods of Information in Medicine 44[2], 2005, 177-181.

[5] S.E. Middleton, J. Herveg, F. Crazzolara, D. Marvin, Y. Poullet, GEMSS: Security and Privacy for a Medical Grid, Methods of Information in Medicine 44[2], 2005, 182-185. 